\title{
Impact of cataract surgery on health-related quality of life in nursing home residents
}

\section{Cynthia Owsley, Gerald McGwin Jr, Kay Scilley, G Christine Meek, Deidre Seker, Allison Dyer}

Br J Ophthalmol 2007;91:1359-1363. doi: 10.1136/bjo.2007.118547

See end of article for authors' affiliations

Correspondence to

Correspondence to:
Cynthia Owsley, Department of Ophthalmology, School of Medicine, University of Alabama at Birmingham, 700 S. 18th Street, Suite 609, Birmingham, AL 35294-0009, USA; owsley@uab.edu

Accepted 3 May 2007 Published Online First 23 May 2007

\begin{abstract}
Aim: To assess the impact of cataract surgery in nursing home residents on health-related quality of life, as compared to those who have cataracts but who do not undergo surgery.

Methods: A prospective cohort study enrolled 30 nursing home residents ( $\geqslant 60$ years old) who had cataracts and underwent cataract surgery, and evaluated vision-targeted and generic health-related quality of life and depressive symptoms before and approximately 4 months after surgery. This cataract surgery group was compared to 15 nursing home residents who had cataracts but who did not have surgery, over the same timeframe.

Results: Visual acuity for near and distance and contrast sensitivity improved following cataract surgery $(p<0.001)$. Adjusting for age differences in the two groups, the cataract surgery group exhibited significant score improvement in the general vision $(p=0.005)$, reading $(p=0.001)$, psychological distress $(p=0.015)$, and social interaction $(p=0.033)$ subscales of the Nursing Home Vision-targeted Health-Related Quality of Life Questionnaire and the VF-14 $(p=0.004)$. There were no group differences in the SF-36, Geriatric Depression Scale or the Cataract Symptom Score.

Conclusion: Nursing home residents who underwent cataract surgery because of functional problems experienced significant improvements in their vision-targeted health-related quality of life, in addition to dramatically improved vision.
\end{abstract}

$\mathrm{N}$ ursing home residents in the USA and other industrialised countries have high rates of vision impairment with estimates ranging from 3 to 15 times higher than corresponding rates for community-dwelling older adults. ${ }^{1-11}$ Cataract is the cause of a substantial portion of bilateral blindness cases among nursing home residents, with studies estimating that cataract is the cause in one-third to two-thirds of blindness cases identified in nursing homes. ${ }^{48}$ Cataract is a highly treatable condition due to dramatic advances in cataract surgery procedures and intraocular lens design. Cataract surgery is safe, highly effective in reversing vision impairment, and a frequent treatment among community-dwelling older adults with it being the most commonly performed surgical procedure among those covered by Medicare. ${ }^{12}$ All these factors raise the question as to why vision impairment from cataracts in nursing home residents is so pervasive. Previous research suggests several contributing causes may underlie this situation, ${ }^{73-17}$ including an under-availability of eye care services in nursing homes, transportation barriers in getting to eye clinics and surgery centres, the attitudes of the residents, their family members/ sponsors, and their healthcare providers that benefits are unlikely given cognitive problems and/or physical frailty, as well as concern and cautiousness on the part of the ophthalmologist that surgery may not be in the best interest of a very elderly patient.

Although there is a very clear and persuasive evidence- basis that cataract surgery enhances quality of life among community-dwelling adults, ${ }^{18-21}$ the question of whether cataract surgery has a positive impact on the quality of life of nursing home residents remains unaddressed. This study is designed to begin to shed light on this question by comparing changes in health-related quality of life in a group of nursing home residents who undergo cataract surgery to a group who also have cataracts but who decline surgery.

\section{PATIENTS AND METHODS}

The institutional review board at the University of Alabama at Birmingham, USA approved the study protocol. Fourteen nursing homes in the Birmingham, Alabama area, USA were sites for the study. All participating nursing homes had eye care services available to residents in that a licensed optometrist visited the facility on a routine basis to provide eye care services to residents, and when the services of an ophthalmologist were needed (eg, evaluation for possible cataract surgery), the optometrist had established referral paths with community ophthalmologists for this purpose. Written informed consent was obtained from all participants and also the resident's sponsor (a family member or state-appointed guardian) after explaining the nature and possible consequences of the study.

Persons were eligible for enrolment if:

- they were identified by the unit charge nurse as a person who could answer simple questions about vision and daily activities;

- they were $\geqslant 55$ years old;

- they spoke English;

- they had a Mini-Mental State Exam (MMSE) $)^{22}$ score of $\geqslant 13$, since comprehension of simple requests and questionnaire items was critical to valid outcomes assessment;

- they had a cataract in one or both eyes that caused functional problems for the resident;

- the optometrist and the ophthalmologist judged that cataract surgery was an appropriate treatment option for one or both eyes given the person's ocular and overall health status.

Residents and their sponsors were informed by the ophthalmologist that cataract surgery was an option in order to improve vision. If the resident and sponsor decided to have surgery on one or both eyes, the surgery was scheduled, and if the resident and sponsor consented to study participation, the resident was assigned to the cataract surgery group. Those who

Abbreviations: NHVQoL, Nursing Home Vision-Targeted Health-Related Quality of Life Questionnaire 
declined cataract surgery and consented to participate were assigned to the no-surgery group. All potential enrolees had health insurance that would cover the cost of cataract surgery and a new spectacle correction following surgery, should they elect surgery. Recruitment for the study proceeded over a twoyear period and was part of a larger study on the visual status of nursing home residents. ${ }^{73}$

The study protocol consisted of a baseline and a follow-up assessment, which were identical. For the surgery group, the baseline assessment was administered before cataract surgery on either eye, and the follow-up assessment was approximately 4 months following surgery after the resident had been using his/her new spectacles for 2-3 months (new spectacles are not typically prescribed until 4-6 weeks following cataract surgery). If both eyes were undergoing surgery, the follow-up assessment was not performed until both eyes underwent surgery. For the no-surgery group, the baseline and follow-up assessments occurred at times that were commensurate with the pre-post duration for the typical surgery enrolee, except of course no cataract surgery had occurred in the intervening period.

Baseline and follow-up assessments were as follows. A research staff member assessed distance and near visual acuity while the resident used habitual correction (or nothing if they used no correction) for each eye separately and together. Testing was carried out either in the resident's room or another private area with adequate lighting. Distance acuity was assessed with the ETDRS chart using its standard protocol and expressed as $\log M A R .{ }^{24}$ Near acuity was assessed using the Lighthouse Near Visual Acuity Test (modified ETDRS) administered at $40 \mathrm{~cm}$. Contrast sensitivity was evaluated using the Pelli-Robson chart ${ }^{25}$ and its standard protocol and scored by the letter-by-letter method. ${ }^{26}$ The Nursing Home Vision-Targeted Health-Related Quality of Life Questionnaire (NHVQoL) ${ }^{27} 28$ was used to assess vision-targeted health-related quality of life. The NHVQoL is an instrument specifically developed for the nursing home resident population and consists of nine subscales focusing on general vision, reading, ocular symptoms, mobility, psychological stress, activities of daily living, activity/ hobbies, adaptation/coping, and social interaction. Two other questionnaires were also administered: the Medical Outcomes Study Short-Form 36 (SF-36) ${ }^{29}$ to assess generic health-related quality of life (both mental and physical components) and the VF-14 to assess the visual activities of daily living. ${ }^{30}$ Scoring on all three questionnaires is from 0 (severe disability) to 100 (no disability), and all questionnaires were interviewer-administered by trained personnel. The presence of depressive symptoms was assessed by the 15-item Geriatric Depression Scale (GDS), ${ }^{31}{ }^{32}$ a widely used screening instrument for estimating depressive symptomatology in older adults including those residing in nursing homes. The Cataract Symptom Score $^{30}$ was obtained by asking residents to what extent they were bothered by five visual symptoms caused by cataract: distorted vision, glare, blurry vision, colours looking different and worsening of vision in the past month. The total score ranging from 0 (not bothered by any symptoms) to 15 (very bothered by all five symptoms) estimates the extent of visual symptomatology. The medical record was abstracted to obtain information on demographic variables (age, gender, race/ ethnicity and education, which were also verified by interview), length of stay in the nursing home, current chronic medical conditions, type of cataract, and characteristics of the surgical procedure if performed.

Statistical $\mathrm{t}$ and $\mathrm{X}^{2}$ tests were used to compare demographic, medical and vision characteristics between the cataract surgery and no-surgery groups. To compare pre-surgery and postsurgery vision among the eyes that underwent surgery a mixed model, which accounted for the correlation of eyes within a
Table 1 Baseline demographic, medical and vision characteristics

\begin{tabular}{|c|c|c|c|}
\hline Variable & $\begin{array}{l}\text { Cataract surgery } \\
\text { group }(n=30)\end{array}$ & $\begin{array}{l}\text { No-surgery } \\
\text { group }(n=15)\end{array}$ & $\mathbf{p}$ \\
\hline Age in years, mean (SD) & $81(8)$ & $87(6)$ & 0.021 \\
\hline \multicolumn{4}{|l|}{ Race/ethnicity, n (\%) } \\
\hline African-American & $7(23.3)$ & $3(20)$ & \multirow[t]{2}{*}{0.800} \\
\hline White, non-Hispanic & $23(77.7)$ & $12(80)$ & \\
\hline \multicolumn{4}{|l|}{ Gender, $n(\%)$} \\
\hline Female & $22(73.3)$ & $13(86.7)$ & \multirow[t]{2}{*}{0.311} \\
\hline Male & $8(26.7)$ & $2(13.3)$ & \\
\hline \multicolumn{4}{|l|}{ Education, n (\%) } \\
\hline $\begin{array}{l}\text { Attended school but not a high school } \\
\text { graduate }\end{array}$ & $19(63.3)$ & $8(53.3)$ & \multirow[t]{3}{*}{0.264} \\
\hline High school graduate & $4(13.3)$ & $5(33.3)$ & \\
\hline At least some college & $7(23.3)$ & $2(13.3)$ & \\
\hline MMSE score, mean (SD) & $21.1(5.3)$ & $19.7(5.3)$ & 0.386 \\
\hline Number of medical conditions, mean (SD) & $6.5(4.0)$ & $4.9(3.8)$ & 0.197 \\
\hline \multicolumn{4}{|l|}{ Cataract type, $n(\%)$} \\
\hline Nuclear sclerotic & $30(100)$ & $13(92.9)$ & 0.139 \\
\hline Cortical & $22(73.3)$ & $10(71.4)$ & 0.895 \\
\hline Posterior subcapsular & $16(53.3)$ & $6(42.9)$ & 0.517 \\
\hline Cataract symptom score & $3.1(3.6)$ & $2.3(4.1)$ & 0.512 \\
\hline \multicolumn{4}{|l|}{ Comorbid eye conditions, n (\%) } \\
\hline Glaucoma & $0(0)$ & $1(6.7)$ & 0.153 \\
\hline Age-related macular degeneration & $3(10)$ & $3(20)$ & 0.352 \\
\hline Diabetic retinopathy & $0(0)$ & $0(0)$ & \\
\hline $\begin{array}{l}\text { Length of nursing home stay in years, mean } \\
\text { (SD) }\end{array}$ & $1.5(1.7)$ & $1.6(1.9)$ & 0.962 \\
\hline \multicolumn{4}{|l|}{ Distance visual acuity in logMAR, mean (SD) } \\
\hline Better eye & $0.56(0.28)$ & $0.53(0.24)$ & 0.761 \\
\hline Worse eye & $0.87(0.25)$ & $0.81(0.29)$ & 0.518 \\
\hline Both eyes & $0.55(0.27)$ & $0.52(0.23)$ & 0.720 \\
\hline \multicolumn{4}{|l|}{ Near visual acuity in logMAR, mean (SD) } \\
\hline Better eye & $0.75(0.29)$ & $0.65(0.23)$ & 0.292 \\
\hline Worse eye & $1.10(0.32)$ & $1.07(0.31)$ & 0.576 \\
\hline Both eyes & $0.72(0.30)$ & $0.63(0.19)$ & 0.326 \\
\hline \multicolumn{4}{|l|}{ Contrast sensitivity, log sensitivity, mean (SD) } \\
\hline Better eye & $0.99(0.40)$ & $0.97(0.32)$ & 0.857 \\
\hline Worse eye & $0.54(0.52)$ & $0.51(0.54)$ & 0.865 \\
\hline Both eyes & $1.03(0.43)$ & $1.07(0.29)$ & 0.729 \\
\hline
\end{tabular}

single patient, was used. Paired t tests were used to compare baseline and follow-up vision, health-related quality of life, depression and cataract symptoms within the surgery and nosurgery groups, whereas analysis of covariance (ANCOVA) was used to compare changes in these characteristics between groups. The dependent variable for these ANCOVA analyses was the follow-up measurement and the primary dependent variable was surgery versus no surgery; the associated baseline measurement for the dependent variable was also included as an independent variable. p Values $\leqslant 0.05$ (two-sided) were considered statistically significant.

\section{RESULTS}

Table 1 displays baseline demographic, medical and vision characteristics for the cataract surgery group $(n=30)$ and the

Table 2 Vision pre-surgery (baseline) and post-cataract surgery (follow-up) for 44 eyes undergoing cataract surgery and intraocular lens insertion eyes in the cataract surgery group

\begin{tabular}{llll}
\hline & $\begin{array}{l}\text { Pre-surgery } \\
\text { (baseline) } \\
\text { mean (SD) }\end{array}$ & $\begin{array}{l}\text { Post-surgery } \\
\text { (follow-up) } \\
\text { mean (SD) }\end{array}$ & P \\
\hline Distance acuity, logMAR & $0.74(0.28)$ & $0.25(0.24)$ & $<0.001$ \\
Near acuity, logMAR & $0.89(0.32)$ & $0.43(0.26)$ & $<0.001$ \\
Contrast sensitivity, log & $0.77(0.51)$ & $1.27(0.24)$ & $<0.001$ \\
sensitivity & & & \\
\hline
\end{tabular}


Table 3 Binocular visual acuity for the surgery and the nosurgery groups at baseline and follow-up

\begin{tabular}{lllll}
\hline & $\begin{array}{l}\text { Baseline } \\
\text { mean (SD) }\end{array}$ & $\begin{array}{l}\text { Follow-up } \\
\text { mean (SD) }\end{array}$ & $\mathbf{p}$ \\
\hline $\begin{array}{l}\text { Surgery group }(n=30) \\
\text { Binocular distance acuity, logMAR }\end{array}$ & $0.55(0.27)$ & $0.23(0.24)$ & $<0.001$ \\
$\quad$ Binocular near acuity, logMAR & $0.72(0.30)$ & $0.38(0.21)$ & $<0.001$ \\
Binocular contrast sensitivity, log & $1.03(0.43)$ & $1.37(.22)$ & $<0.001$ \\
sensitivity & & & \\
No-surgery group ( $=15)$ & & & \\
$\quad$ Binocular distance acuity, logMAR & $0.52(0.23)$ & $0.59(0.29)$ & 0.170 \\
$\quad$ Binocular near acuity, logMAR & $0.63(0.19)$ & $0.68(0.27)$ & 0.368 \\
$\quad$ Binocular contrast sensitivity, log & $1.07(0.29)$ & $0.87(0.47)$ & 0.094 \\
sensitivity & & & \\
\hline
\end{tabular}

no-surgery group $(n=15)$. The cataract surgery group was younger on average than was the no-surgery group. The groups were not different with respect to gender, race/ethnicity, education, mental status, number of medical comorbidities, type of cataract, cataract symptom score or length of stay in the nursing home. Baseline visual function (distance and near acuity, contrast sensitivity) was also similarly distributed in the two groups, whether analysed by the better eye, worse eye, or by vision using both eyes.

All cataract surgery was performed on an outpatient basis. Of the 30 residents who had cataract surgery, nine were transported to the surgery centre by the family, 15 by a van paid for by health insurance or the nursing home, and six by a van paid for by the project. The most common functional problems prompting cataract surgery were difficulties with reading, watching TV and seeing in the distance, cited by over $90 \%$ of those electing surgery. Phacomulsification was used on $80 \%$ of eyes, with $20 \%$ of eyes undergoing extracapsular extraction. Posterior chamber intraocular lenses were implanted in all but one eye that received an anterior chamber lens. YAG capsulotomy was performed on 15 of 44 eyes. Only one eye had a perioperative complication (vitreous loss), and two eyes had postoperative complications (cystoid macular oedema, posterior capsular fibrosis). With respect to visual function improvement from baseline to follow-up on the 44 operated eyes (table 2), distance acuity improved on average from approximately $20 / 100$ to $20 / 35$, near acuity improved from approximately $20 / 150$ to $20 / 50$, and contrast sensitivity improved by $1 / 2 \log$ unit.

The use of both eyes together is how participants experience the world and thus it is useful to examine how visual function under binocular viewing changed from baseline to follow-up in both groups. Table 3 shows that distance acuity, near acuity and contrast sensitivity under binocular viewing improved significantly in the cataract surgery group from baseline to follow-up, whereas these aspects of vision remained unchanged in the no-surgery group over this same time period.

Table 4 displays the outcomes assessment. For the cataract surgery group, all subscales of the NHVQoL had improved scores following cataract surgery, as did the VF-14. GDS scores decreased on average at follow-up as did the cataract symptom score (signifying fewer symptoms in these domains). The physical and mental component scores of the SF-36 were unchanged. None of the outcome measures changed in the nosurgery group from baseline to follow-up. In comparing the change in scores in the two groups from baseline to follow-up, after adjusting for age differences in the two groups, the cataract surgery group exhibited significant score improvements in the general vision, reading, psychological distress and social interaction subscales of the NHVQoL and in the VF-14. There were no differences between the surgery and no-surgery groups with respect to changes in the SF-36, GDS or the cataract symptom score.

Table 4 Outcome: Health-related quality of life, depression, and cataract symptom list scores

\begin{tabular}{|c|c|c|c|c|c|c|c|c|}
\hline \multirow[b]{2}{*}{ Variable } & \multicolumn{3}{|c|}{ Cataract surgery group $(n=30)$} & \multicolumn{3}{|c|}{ No-surgery group ( $n=15$ ) } & \multirow[b]{2}{*}{$p^{*}$} & \multirow[b]{2}{*}{ pt } \\
\hline & Baseline & Follow-up & $\mathbf{p}$ & Baseline & Follow-up & $\mathbf{p}$ & & \\
\hline \multicolumn{9}{|l|}{ NHVQoL, mean (SD) } \\
\hline General vision & $\begin{array}{l}57.2 \\
(23.5)\end{array}$ & $\begin{array}{l}79.3 \\
(15.8)\end{array}$ & $<0.001$ & $\begin{array}{l}65.7 \\
(22.9)\end{array}$ & $\begin{array}{l}67.7 \\
(21.1)\end{array}$ & 0.653 & 0.014 & 0.005 \\
\hline Reading & $\begin{array}{l}69.4 \\
(27.5)\end{array}$ & $\begin{array}{l}93.6 \\
(12.1)\end{array}$ & $<0.001$ & $\begin{array}{l}78.3 \\
(26.3)\end{array}$ & $\begin{array}{l}78.3 \\
(22.2)\end{array}$ & 0.999 & 0.001 & 0.001 \\
\hline Ocular symptoms & $\begin{array}{l}70.3 \\
(29.8)\end{array}$ & $\begin{array}{l}86.3 \\
(20.3)\end{array}$ & $<0.001$ & $\begin{array}{l}69.8 \\
(32.1)\end{array}$ & $\begin{array}{l}81.0 \\
(27.2)\end{array}$ & 0.224 & 0.378 & 0.288 \\
\hline Mobility & $\begin{array}{l}82.9 \\
(18.2)\end{array}$ & $\begin{array}{l}93.5 \\
(5.6)\end{array}$ & 0.008 & $\begin{array}{l}90.2 \\
(12.2)\end{array}$ & $\begin{array}{l}91.9 \\
(9.8)\end{array}$ & 0.259 & 0.511 & 0.323 \\
\hline Psychological distress & $\begin{array}{l}62.0 \\
(27.0)\end{array}$ & $\begin{array}{l}77.7 \\
(15.1)\end{array}$ & 0.001 & $\begin{array}{l}72.0 \\
(17.3)\end{array}$ & $\begin{array}{l}68.4 \\
(24.3)\end{array}$ & 0.570 & 0.087 & 0.015 \\
\hline Activities of daily living & $\begin{array}{l}92.5 \\
(15.8)\end{array}$ & $\begin{array}{l}97.1 \\
(9.3)\end{array}$ & 0.128 & $\begin{array}{l}95.0 \\
(19 . .4)\end{array}$ & $\begin{array}{l}94.2 \\
(19.4)\end{array}$ & 0.334 & 0.379 & 0.349 \\
\hline Activities and hobbies & $\begin{array}{l}85.2 \\
(20.6)\end{array}$ & $\begin{array}{l}95.9 \\
(11.9)\end{array}$ & 0.001 & $\begin{array}{l}95.4 \\
(7.4)\end{array}$ & $\begin{array}{l}88.2 \\
(24.4)\end{array}$ & 0.171 & 0.142 & 0.083 \\
\hline Adaptation and coping & $\begin{array}{l}83.8 \\
(26.9)\end{array}$ & $\begin{array}{l}93.3 \\
(14.6)\end{array}$ & 0.026 & $\begin{array}{l}86.7 \\
(18.6)\end{array}$ & $\begin{array}{l}85.8 \\
(30.2)\end{array}$ & 0.931 & 0.254 & 0.118 \\
\hline Social interaction & $\begin{array}{l}86.4 \\
(20.1)\end{array}$ & $\begin{array}{l}98.1 \\
(5.8)\end{array}$ & 0.004 & $\begin{array}{l}94.2 \\
(9.5)\end{array}$ & $\begin{array}{l}91.2 \\
(17.4)\end{array}$ & 0.282 & 0.048 & 0.033 \\
\hline VF-14, mean (SD) & $\begin{array}{l}68.7 \\
(23.5)\end{array}$ & $\begin{array}{l}93.6 \\
(14.2)\end{array}$ & $<0.001$ & $\begin{array}{l}80.5 \\
(24.3)\end{array}$ & $\begin{array}{l}82.0 \\
(23.2)\end{array}$ & 0.648 & 0.015 & 0.004 \\
\hline \multicolumn{9}{|l|}{ SF-36, mean (SD) } \\
\hline Mental component summary & $\begin{array}{l}81.2 \\
(16.1)\end{array}$ & $\begin{array}{l}85.3 \\
(13.5)\end{array}$ & 0.224 & $\begin{array}{l}82.1 \\
(11.1)\end{array}$ & $\begin{array}{l}86.5 \\
(7.0)\end{array}$ & 0.234 & 0.851 & 0.949 \\
\hline Physical component summary & $\begin{array}{l}45.9 \\
(14.4)\end{array}$ & $\begin{array}{l}44.0 \\
(14.2)\end{array}$ & 0.523 & $\begin{array}{l}45.9 \\
(15.8)\end{array}$ & $\begin{array}{l}45.6 \\
(14.3)\end{array}$ & 0.897 & 0.745 & 0.914 \\
\hline GDS, mean (SD) & $\begin{array}{r}4.4 \\
(2.6)\end{array}$ & $\begin{array}{r}3.9 \\
(2.9)\end{array}$ & 0.003 & $\begin{array}{r}4.1 \\
(3.5)\end{array}$ & $\begin{array}{r}3.4 \\
(2.7)\end{array}$ & 0.462 & 0.908 & 0.951 \\
\hline Cataract symptom score, mean (SD) & $\begin{array}{r}3.1 \\
(3.6)\end{array}$ & $\begin{array}{r}1.1 \\
(2.6)\end{array}$ & 0.002 & $\begin{array}{r}2.3 \\
(4.1)\end{array}$ & $\begin{array}{r}1.7 \\
(2.6)\end{array}$ & 0.513 & 0.522 & 0.840 \\
\hline
\end{tabular}

${ }^{*}$ Comparison of between-group changes from baseline to follow-up. †Comparison of between-group changes from baseline to follow-up, adjusted for age. 


\section{DISCUSSION}

Nursing home residents who underwent cataract surgery because of functional problems experienced short-term significant improvements in their vision-targeted health-related quality of life, in addition to improvements in their vision. In contrast, nursing home residents who had cataracts but who did not undergo surgery did not exhibit these improvements. Following cataract surgery residents reported improved general vision, fewer limitations because of their vision, less difficulty with reading, less worry, frustration and/or upset over their vision, and greater ease and likelihood of engaging in social interactions. These quality-of-life benefits are not surprising given the dramatic improvement in visual acuity and contrast sensitivity that ensued following surgery. For example, distance acuity was seriously impaired, averaging $20 / 100$ prior to surgery and after surgery was 20/35 on average.

It is important to emphasise that the characteristics of the surgical care process for these nursing home residents were highly similar to those of people residing in the community who undergo cataract surgery. Surgery was performed on an outpatient basis, primarily involved the use of posterior chamber intraocular lenses, had a very low complication rate, was covered by health insurance and had excellent visual outcomes. ${ }^{12}$ Although the sample size of surgical cases was small in this study $(n=30)$, our results are consistent with the notion that the surgical practices that ophthalmologists use when caring for community-dwelling older adults with cataracts may be extended with success into the nursing home population.

The results of this study contribute to the growing body of evidence that older nursing home residents can benefit from eye care interventions to improve visual function. We have previously shown that refractive error correction (ie, spectacles to correct near-sightedness, far-sightedness, presbyopia) enhances vision-targeted health-related quality of life, reduces difficulty in the visual activities of daily living and decreases depressive symptomatology in nursing home residents. ${ }^{23}$ Teresi and colleagues demonstrated that refractive error correction in nursing home residents slowed functional decline (ability to independently perform activities of daily living, range of motion, walking ability) over a 6-8-month period; when refractive error correction was combined with a nursing assistant training programme to recognise vision impairment and reinforce the use of spectacles in residents, there was an increase in function. ${ }^{33}$

Unlike our previous finding that refractive error correction decreased depression symptomatology in nursing home residents, ${ }^{23}$ we found no analogous impact for cataract surgery. While depressive symptoms within the cataract surgery group decreased following surgery, this decrease was not different from that observed in the no-surgery group, although the small sample size in this study may have engendered inadequate statistical power for evaluating this outcome. The lack of association between cataract surgery and depression finding in this nursing home resident sample is consistent with our previous work on community-dwelling older adults showing that depressive symptoms do not decrease following cataract surgery when compared with a group of older adults with cataracts who do not undergo surgery. ${ }^{34}$

Even though our vision-targeted health-related quality of life instrument revealed improvement, the physical and mental component scores of the SF-36 did not improve following cataract surgery, suggesting that a generic measure of healthrelated quality of life may not be sensitive enough to the condition-specific or function-specific gains that ensue after cataract surgery, a possibility noted in the literature previously. ${ }^{35}$
A strength of this study is the inclusion of a comparison group of nursing home residents with cataracts who did not undergo surgery and were followed over the same time period. In addition, primary outcome measures selected for the study were valid and reliable tools for assessment of their respective constructs. Limitations must also be acknowledged. Since cataract surgery followed by intraocular lens implantation is a standard of care for the treatment of cataract, a randomised design where residents are assigned to surgery or no-surgery was not ethically possible. Thus, any observed differences in the outcomes measures of interest may be due to differences in demographic, medical or other characteristics between the surgery and no-surgery groups. To evaluate this, we compared the surgery and no-surgery groups at baseline with respect to a number of potentially confounding factors (see table 1). The only variable that differed between the two groups was age, which was adjusted for in comparison of outcomes between the two groups. The sample size was small, although in spite of this, positive findings were obtained on some outcomes of interest. This study was not designed to examine the long-term efficacy of cataract surgery. In addition, it cannot address the efficacy of cataract surgery for nursing home residents with MMSE scores below 13. This is an issue worthy of further investigation since severe cognitive impairment is not uncommon in the nursing home population.

With mounting evidence that eye care interventions in the nursing home, such as cataract surgery and refractive error correction, improve health-related quality of life, there is a sound rationale to identify the causes of under-provision of eye care services to nursing home residents, ${ }^{13-16}$ and then once identified, to develop strategies to overcome these barriers to care. At least half of nursing home residents in the USA live at least one or more years in the nursing home and $21 \%$ reside there for almost 5 years. ${ }^{36}$ These are lengthy time periods for a population to be without even the most basic of eye care services given their high risk for vision impairment. ${ }^{1-11}$

\section{Authors' affiliations \\ Cynthia Owsley, Gerald McGwin Jr, Kay Scilley, G Christine Meek, Deidre Seker, Allison Dyer, Department of Ophthalmology, University of Alabama at Birmingham \\ Gerald McGwin Jr, Department of Epidemiology and International Health, University of Alabama at Birmingham \\ Gerald McGwin Jr, Department of Surgery, University of Alabama at Birmingham}

Funding: This research was supported by the Retirement Research Foundation; the EyeSight Foundation of Alabama; the Pearle Vision Foundation; National Institutes of Health grant R21-EY14071; and Research to Prevent Blindness, Inc. CO is a Research to Prevent Blindness Senior Scientific Investigator.

Competing interests: None declared.

\section{REFERENCES}

1 Whitmore WG. Eye disease in a geriatric nursing home population. Ophthalmology 1989;96:393-8.

2 Klein R, Klein BEK, Linton KLP, et al. The Beaver Dam Eye Study: visual acuity. Ophthalmology 1991;98:1310-5.

3 Horowitz A. Vision impairment and functional disability among nursing home residents. Gerontologist 1994;34:316-23.

4 Tielsch JM, Javitt JC, Coleman A, et al. The prevalence of blindness and visual impairment among nursing home residents in Baltimore. N Engl J Med 1995;332:1205-9.

5 West SK, Friedman D, Munoz B, et al. A randomized trial of visual impairment interventions for nursing home residents: study design, baseline, characteristics, and visual loss. Ophthalmic Epidemiol 2003;10:193-209.

6 Friedman DS, West SK, Munoz B, et al. Racial variations in causes of vision loss in nursing homes. Arch Ophthalmol 2004;122:1019-24.

7 Owsley C, McGwin Jr G, Scilley K, et al. The visual status of older persons residing in nursing homes. Arch Ophthalmol 2007; 125:925-30.

8 Mitchell P, Hayes P, Wang JJ. Visual impairment in nursing home residents: The Blue Mountains Eye Study. Med J Aust 1997;166:73-6. 
9 Taiel-Sartral M, Nounou P, Rea C, et al. Visual acuity and ocular disease in geriatric nursing homes. Study in 219 elderly people in France. J Fr Ophtalmol 1999:22:431-7.

10 VanNewkirk MR, Weih L, McCarty CA, et al. Visual impairment and eye diseases in elderly institutionalized Australians. Ophthalmology 2000;107:2203-8.

11 de Winter LJ, Hoyng CB, Froeling PG, et al. Prevalence of remediable disability due to low vision among institutionalised elderly people. Gerontology 2004;50:96-101.

12 Powe NR, Schein OD, Gieser SC, et al. Synthesis of the literature on visual acuity and complications following cataract extraction with intraocular lens implantation. Arch Ophthalmol 1994;1 12:239-52.

13 National Center for Health Statistics. National nursing home survey. http:// www.cdc.gov/nchs/nnhs.htm (accessed 16 Nov 2006).

14 Agency for Healthcare Research and Quality. Medical expenditure panel survey. http://www.meps.ahrq.gov/mepsweb/ (accessed 16 Nov 2006).

15 Voytas JJ, Kowalski D, Wagner S, et al. Eye care in the skilled nursing facility: a pilot study of prevalence and treatment patterns of glaucoma. J Am Med Dir Assoc 2004;5:156-60.

16 Keller BK, Hejkal T, Potter JF. Barriers to vision care for nursing home residents. J Am Med Dir Assoc 2001;2:15-21.

17 Friedman DS, Munoz B, Roche KB, et al. Poor uptake of cataract surgery in nursing home residents. The Salisbury Eye Evaluation in Nursing Home Groups Study. Arch Ophthalmol 2005;123:1581-7.

18 Steinberg EP, Tielsch JM, Schein OD, et al. National study of cataract surgery outcomes: variation in 4-month postoperative outcomes as reflected in multiple outcome measures. Ophthalmology 1994;101:1131-41.

19 Mangione CM, Phillips RS, Lawrence MG, et al. Improved visual function and attenuation of declines in health-related quality of life after cataract extraction. Arch Ophthalmol 1994;112:1419-25.

20 Javitt JC, Brenner MH, Curbow B, et al. Outcomes of cataract surgery: improvement in visual acuity and subjective visual function after surgery in the first, second, and both eyes. Arch Ophthalmol 1993;111:686-91.

21 Owsley C, McGwin Jr G, Sloane ME, et al. Impact of cataract surgery on motor vehicle crash involvement by older adults. JAMA 2002;288:841-9.
22 Folstein MF, Folstein SW, McHugh PR. "Mini-mental state": a practical method for grading the cognitive state of patients for the clinician. J Psychiatr Res 1975;12:189-98.

23 Owsley C, McGwin Jr G, Scilley K, et al. Effect of refractive error correction on health-related quality of life and depression in older nursing home residents. Arch Ophthalmol 2007 (in press).

24 Ferris FL, Kassoff A, Bresnick GH, et al. New visual acuity charts for clinical research. Am J Ophthalmol 1982;94:91-6.

25 Pelli DG, Robson JG, Wilkins AJ. The design of a new letter chart for measuring contrast sensitivity. Clin Vision Sci 1988;2:187-99.

26 Elliott DB, Bullimore MA, Bailey IL. Improving the reliability of the Pelli-Robson contrast sensitivity test. Clin Vision Sci 1991;6:471-5.

27 Dreer LE, Scilley K, McGwin Jr G, et al. Development of a nursing home visiontargeted health-related quality of life questionnaire. Aging Ment Health 2007 (in press).

28 Scilley K, Owsley C. Vision-specific health-related quality of life: content areas for nursing home residents. Qual Life Res 2002;11:449-62.

29 Ware Jr J, Sherbourne CD. The MOS 36-item short-form health survey (SF-36): I. Conceptual framework and item selection. Med Care 1992;30:473-83.

30 Steinberg EP, Tielsch JM, Schein OD, et al. The VF-14: an index of functional impairment in patients with cataract. Arch Ophthalmol 1994;112:630-8.

31 Yesavage JA, Brink TL, Rose TL, et al. Development and validation of a geriatric depression screening scale: a preliminary report. J Psychiatr Res 1982;17:37-49.

32 Sheikh JI, Yesavage JA. Geriatric Depression Scale (GDS): Recent evidence and development of a shorter version. In: Brink TL, eds. Clinical gerontology: a guide to assessment and intervention. New York: Haworth Press, 1986:165-73.

33 Teresi J, Morse A, Holmes D, et al. Impact of a vision intervention on the functional status of nursing home residents. J Vis Imp Blind 2005;99:96-108.

34 McGwin Jr G, Li J, McNeal SF, et al. The impact of cataract surgery on depression among older adults. Ophthalmic Epidemiol 2003;10:303-13.

35 Wiebe S, Guyatt G, Weaver B, et al. Comparative responsiveness of generic and specific quality-of-life instruments. J Clin Epidemiol 2003;56:52-60.

36 The AGS Foundation for Health in Aging. Nursing home care. http:// www.healthinaging.org/agingintheknow/ (accessed 11 Dec 2006).

\section{Let us assist you in teaching the next generation}

Figures from all articles on our website can be downloaded as a PowerPoint slide. This feature is ideal for teaching and saves you valuable time. Just click on the image you need and choose the "PowerPoint Slide for Teaching" option. Save the slide to your hard drive and it is ready to go. This innovative function is an important aid to any clinician, and is completely free to subscribers. (Usual copyright conditions apply.) 